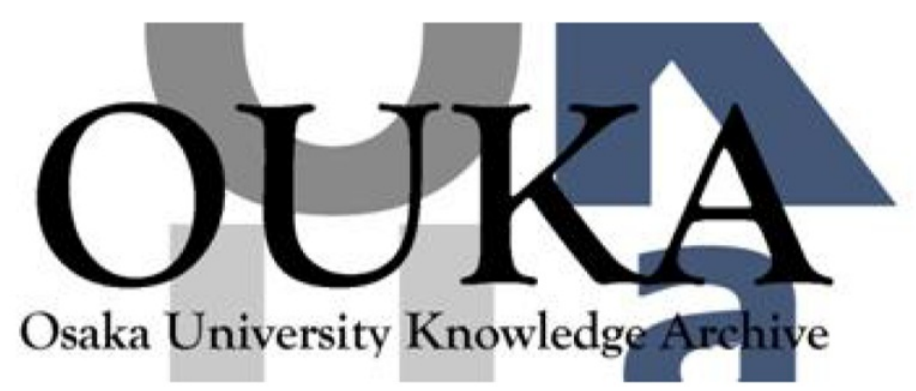

\begin{tabular}{|c|c|}
\hline Title & $\begin{array}{l}\text { Robot-on-Robot Gossiping to Improve Sense of } \\
\text { Human-Robot Conversation }\end{array}$ \\
\hline Author (s) & $\begin{array}{l}\text { Mitsuno, Seiya; Yoshikawa, Yuichiro; Ishiguro, } \\
\text { Hiroshi }\end{array}$ \\
\hline Citation & $\begin{array}{l}\text { 29th IEEE International Conference on Robot and } \\
\text { Human Interact ive Communication, R0-MAN } 2020 \text {. } \\
\text { p. } 653-\text { p. } 658\end{array}$ \\
\hline Issue Date & $2020-08$ \\
\hline oaire:version & AM \\
\hline URL & https://hdl. handle. net/11094/78296 \\
\hline rights & $\begin{array}{l}\text { (- } 2020 \text { IEEE. Personal use of this material is } \\
\text { permitted. Permission from IEEE must be } \\
\text { obtained for all other uses, in any current or } \\
\text { future media, including reprinting/republishing } \\
\text { this material for advertising or promotional } \\
\text { purposes, creating new collective works, for } \\
\text { resale or redistribution to servers or lists, } \\
\text { or reuse of any copyrighted component of this } \\
\text { work in other works. }\end{array}$ \\
\hline Note & \\
\hline
\end{tabular}

Osaka University Knowledge Archive : OUKA

https://ir. Library. osaka-u. ac. jp/

Osaka University 


\title{
Robot-on-Robot Gossiping to Improve Sense of Human-Robot Conversation
}

\author{
Seiya Mitsuno, Yuichiro Yoshikawa, and Hiroshi Ishiguro ${ }^{1}$
}

\begin{abstract}
In recent years, a substantial amount of research has been aimed at realizing a social robot that can maintain long-term user interest. One approach is using a dialogue strategy in which the robot makes a remark based on previous dialogues with users. However, privacy problems may occur owing to private information of the user being mentioned. We propose a novel dialogue strategy whereby a robot mentions another robot in the form of gossiping. This dialogue strategy can improve the sense of conversation, which results in increased interest while avoiding the privacy issue. We examined our proposal by conducting a conversation experiment evaluated by subject impressions. The results demonstrated that the proposed method could help the robot to obtain higher evaluations. In particular, the perceived mind was improved in the Likert scale evaluation, whereas the robot empathy and intention to use were improved in the binary comparison evaluation. Our dialogue strategy may contribute to understanding the factors regarding the sense of conversation, thereby adding value to the field of human-robot interaction.
\end{abstract}

\section{INTRODUCTION}

In recent years, substantial research has been conducted on social robots relating to communication [1]-[3]. Communication with robots has attracted increased attention not only in the service industry for the purpose of entertaining users [1], but also welfare fields, such as communication support for the elderly [2] as well as education, such as language learning [3]. Moreover, several researchers have particularly focused on the exchange of subjective opinions in humanrobot conversations [4], [5]. However, many researchers have confirmed that, when conversational robots are introduced into a real environment over a long period, users gradually lose interest in the robots and the number of interactions decreases with time [1], [3], [6]-[8]. In this study, we aim to realize a conversational robot that can maintain the user's willingness to talk by improving the sense of conversation between humans and robots.

One approach for improving the sense of conversation is the use of a dialogue strategy, in which the robot makes remarks based on previous dialogues with users. Attempts have been made to implement a dialogue strategy in which the robot changes the content of its speech when addressing a user with whom it has previously conversed; for example, "Thank you for coming to see me again!" [9] and "This is the third time you have ordered snickers" [10]. This dialogue strategy can make the user perceive that the robot remembers the past interactions. Other proposals have used

\footnotetext{
${ }^{1}$ Seiya Mitsuno, Yuichiro Yoshikawa and Hiroshi Ishiguro are with Intelligent Robotics Lab, Department of Systems Innovation, Graduate School of Engineering Science, Osaka University. \{mitsuno. seiya, Yoshikawa, ishiguro\}eirl.sys.es.osaka-u.ac.jp
}

a dialogue strategy in which the robot refers to a user's past remark in conversation with another user as gossiping, such as "The previous guy told me he likes red" [11], [12]. In summary, dialogue strategies involving remarks based on past conversation experiences can imply the robot's memory ability [9], [10], sociability [11] and perceived mind [12] to the user.

However, in the current information society where the awareness of privacy is increasing [13], a strong probability exists that privacy problems will occur when the robot mentions the user information (including preferences, past behaviors, and names), which causes the user to feel uncomfortable. In a dialogue strategy in which a robot refers to a user's past remarks in conversation with another user, namely gossiping [11], [12], a user whose private information is exposed by the robot may feel discomfort. Moreover, a privacy issue may arise even in a dialogue strategy where the robot talks about past dialogue experiences with the exact user who is currently interacting with it [9], [10]. This is because, in real-world applications, the robot is likely to face situations where its remarks about user information are heard not only by the currently interacting user, but also by the people around him/her. Moreover, in facilities such as shopping malls and museums, where people tend to socialize in groups, we assume that robots interact with multiple users simultaneously, and in this particular situation, it is more likely that the robot will expose the private information of a specific user. A possible solution to this is to determine which information can be mentioned in subsequent conversations. However, the conversation would have to be interrupted to ask for consent every time new information is provided. Thus, such a solution to the privacy issue may deteriorate the tempo of the conversation, thereby reducing the sense of conversation. Furthermore, it is not easy to form a sufficient agreement with the user, because whether the user agrees to the exposure of his/her private information depends not only on the content of the information but also on the person to whom the information is mentioned and the situation.

Our proposal is to replace users with robots as targets of gossiping to maintain the increased sense of conversation but avoid the privacy issue. Here, we define gossiping as referring to actors not involved in the conversation. As the effect of robot-on-robot gossiping has not yet been investigated, we verify the influence thereof on the sense of conversation.

In this study, we deal with the situation in which a user and a robot are exchanging their subjective opinions. When the opinions do not match, the robot mentions another robot 
with the same opinion as the user. We selected this situation for the following reason. Humans react negatively to the frequent occurrence of disagreements in conversation [5], [14]. In a human-robot dialogue in which a robot has several subjective opinions and preferences, if the robot expresses its own opinions, sometimes the robot will not agree with humans. In such a situation, it is difficult to state simply that the robot should change its own opinion appropriately for each user. This is because, when a robot is supposed to interact with various users in a community, if the robot changes its own opinion for each user, there is a significant possibility that the user will notice the inconsistency of the robot opinion. For example, a lack of consistency in the robot remarks may appear in situations such as when users share the robot's remarks in daily conversation, or when the robot talks to another user in a place where there is a user who has had a conversation with the robot in the past. If the user recognizes the robot's opinions as inconsistent, the reliability of the robot remarks and the attribution of the robot opinions are significantly decreased, which may negatively affect the user's willingness to talk and the sense of conversation. Therefore, there is a need for a dialogue strategy that can mitigate the user's negative reaction when disagreements between humans and robots are unavoidable while exchanging subjective opinions. We believe that the robot can indirectly demonstrate empathy for the user's opinion while maintaining the consistency of its own opinion by referring to another robot with the same opinion as the user, which can reduce the negative human reactions caused by disagreements.

In conclusion, this study aims to verify whether a robot's remark regarding another robot can improve the sense of conversation when the robot cannot agree with the user. The remainder of this paper is organized as follows. We present the experiment and results in Section II, provide a general discussion in Section III, and offer concluding remarks in Section IV.

\section{EXPERIMENT}

To verify the effect of our dialogue strategy, we conducted an experiment with a within-subject design to compare a dialogue with the mention of another robot (social condition) and without (non-social condition).

\section{A. Subjects}

A total of 25 students with a university education background who were fluent in Japanese (13 males and 12 females, ranging in age from 18 to 27) participated in the experiment. Among these, 14 subjects ( 7 males and 7 females) engaged in the social condition dialogue first, and the remaining 11 subjects (5 males and 6 females) engaged in the non-social condition dialogue first.

\section{B. Apparatus}

Robot: We used CommU, a small child-like robot, for the experiment [15] (Fig. 1). This robot possesses 14 degrees of freedom (three for the eyeballs, three for the neck, one for the mouth, one for the eyelids, two for each arm, and two for the waist). Its height is approximately $30 \mathrm{~cm}$, and it has a speaker inside its chest.

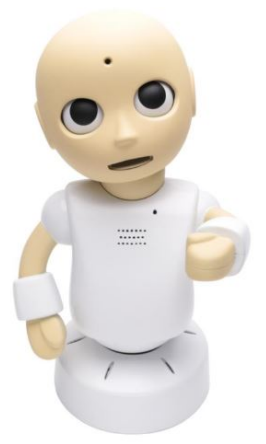

Fig. 1. CommU

In the experiment, we changed the voice and appearance of the robot in the first and second dialogues to create the impression that the subject was talking to a different robot. In this manner, we could remove the influence of prior impressions of the robot. To vary the robot appearance, we changed the hat and nameplate on the chest each time.

System: We used a scenario-based dialogue system in the experiment. In such a system, the robot makes statements or gestures based on the scenario. Moreover, the robot can change its responses depending on the subject utterance. The experimenter is invisible to the subject and controls the scenario transition based on the subject utterances to prevent dialogue breakdown owing to the failure of voice recognition.

Dialogue scenario: Fig. 2 presents the dialogue flows adopted in the two different scenarios. Firstly, we explain the flow for the social condition (Fig. 2(a)). The robot greets the subject and introduces itself (Intro). It then asks the subject about food preferences (R:Question). If his/her food preferences match those pre-registered in the robot, it agrees with the subject's opinion (R:Agree); if not, it disagrees (R:Disagree). If the robot disagrees with the subject's opinion, it shows concern, such as "Oh, but don't worry" (R:Show concern). Thereafter, it mentions another robot with the same opinion as the user (R:Mention another robot). Table I and Table II display example conversations of the social and nonsocial conditions respectively. In Table I, "[another robot name]" indicates the name of the robot to be mentioned. Note that the existence of other robots to be mentioned is introduced to the subject in advance, prior to beginning the dialogue. The robot to be mentioned is selected from two robots. We set $n$, which indicates the number of loops, to 6 in both conditions. Furthermore, the specific dialogue content is food preference.

Secondly, we explain the flow for the non-social condition (Fig. 2(b)). The condition is the same as the social condition, except that when the robot disagrees with the user (R:Disagree), it only shows concern (R:Show concern) and it does not mention another robot. For this reason, the sentence in the final column in Table I, that is, "[another robot name] told me he likes cake, same as you", does not exist in Table II. 
The three robots (robots $\mathrm{A}, \mathrm{B}$, and $\mathrm{C}$ ) that can enter the dialogue have registered food preferences for three different taste categories, namely sweet, sour and spicy (Table III). In the experiment, robot A talked to the subject in the first dialogue, whereas robot $\mathrm{B}$ talked in the second dialogue.

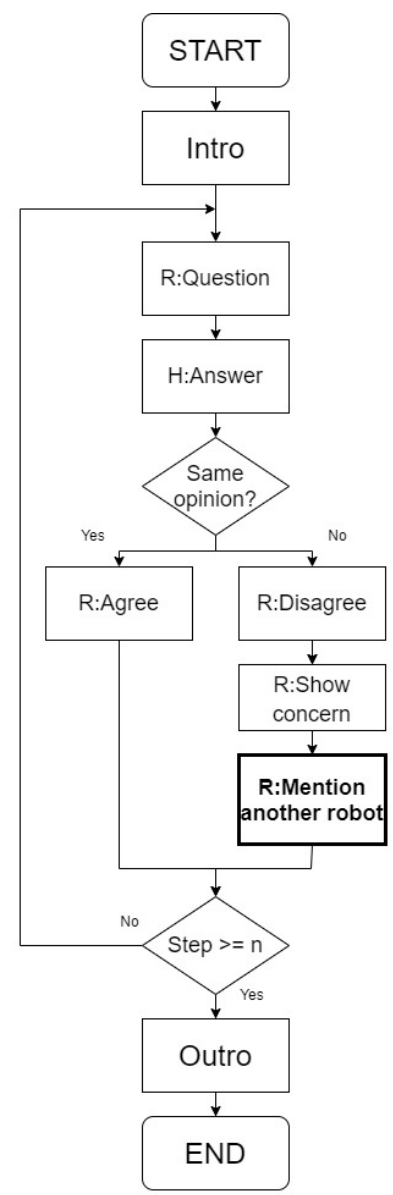

(a) Social condition

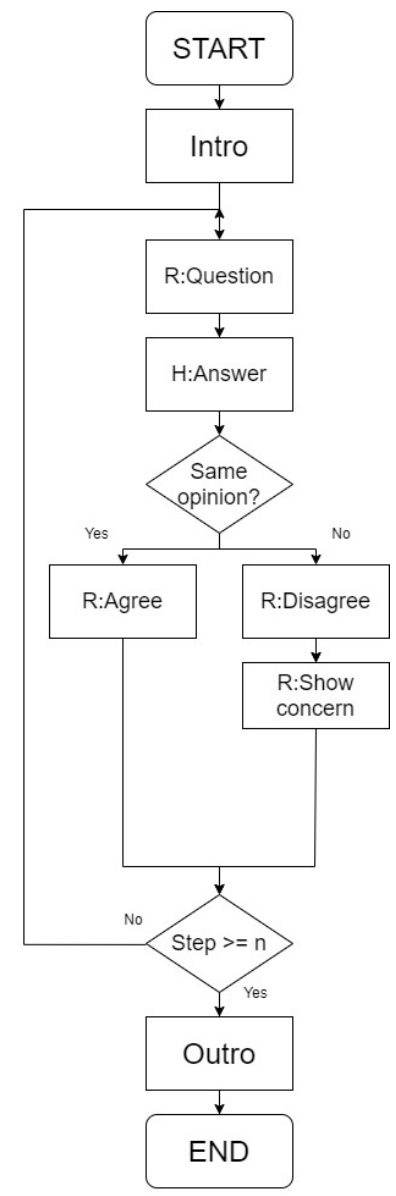

(b) Non-social condition
Fig. 2. Dialogue flows for (a) social condition and (b) non-social condition. R: robot; H: human

TABLE I

EXAMPLE CONVERSATION (SOCIAL CONDITION)

\begin{tabular}{lll}
\hline & In case of agreement & In case of disagreement \\
\hline Robot & Well, do you like cake? & Well, do you like cake? \\
User & Yes, I do. & Yes, I do. \\
Robot & Oh, really? I like cake too & I see. I don't like cake be- \\
& because it's sweet. & cause it's sweet. \\
Robot & & Oh, but don't worry. \\
Robot & & [another robot name] told \\
& & me he likes cake, same as \\
& & you. \\
\hline
\end{tabular}

\section{Procedure}

Following informed consent, the experimenter provided instructions regarding the experiment to each subject. The experimenter told the subject to have a verbal conversation with the robot. The subject was required not to provide
TABLE II

EXAMPLE CONVERSATION (NON-SOCIAL CONDITION)

\begin{tabular}{lll}
\hline & In case of agreement & In case of disagreement \\
\hline Robot & Well, do you like cake? & Well, do you like cake? \\
User & Yes, I do. & Yes, I do. \\
Robot & Oh, really? I like cake too & I see. I don't like cake be- \\
& because it's sweet. & cause it's sweet. \\
Robot & & Oh, but don't worry. \\
\hline
\end{tabular}

TABLE III

FOOD PREFERENCES LIST

\begin{tabular}{cccc}
\hline & Robot A & Robot B & Robot C \\
\hline Sweet & Like & Dislike & Dislike \\
Sour & Dislike & Like & Dislike \\
Spicy & Dislike & Dislike & Like \\
\hline
\end{tabular}

any neutral or ambiguous answers such as "Neither" when the robot asked a question about food preferences. After receiving the instructions, the subject was asked to read the questionnaire used for the experiment. Furthermore, to familiarize the subject with the robots possibly mentioned in the first dialogue, the experimenter showed the subject a photograph of the three robots with their names (Fig. 3). Thereafter, the subject was asked to have a conversation with the robot. When the first conversation was finished, the experimenter asked the subject to fill in a questionnaire about the dialogue. While the subject was completing the questionnaire, the experimenter prepared the second dialogue by changing the robot's voice and appearance. The experimenter then showed the subject another photograph of the three robots that could appear in the second dialogue (Fig. 4), and asked the subject to have a conversation with the robot and fill in a questionnaire about the second dialogue. Finally, the experimenter asked the subject to complete another questionnaire which is a binary comparison of the two dialogues. Fig. 5 presents the scene of the experiment.

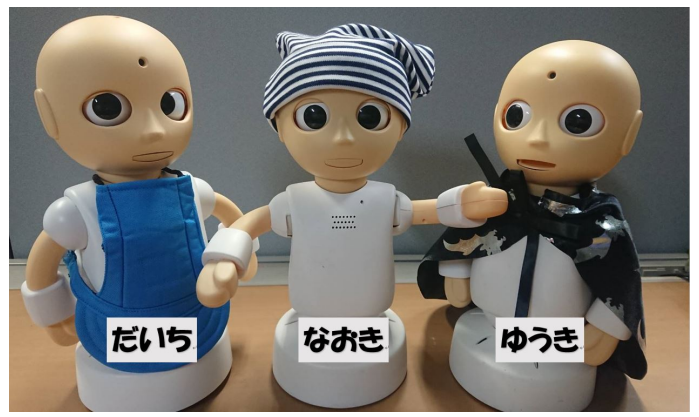

Fig. 3. Photograph shown to subject before first dialogue (each robot's name is displayed at the bottom)

\section{Measurements}

In this section, we detail the question items used to investigate whether the robot could improve the sense of conversation by mentioning another robot. We conducted the evaluation using four measurements. As factors for the sense 


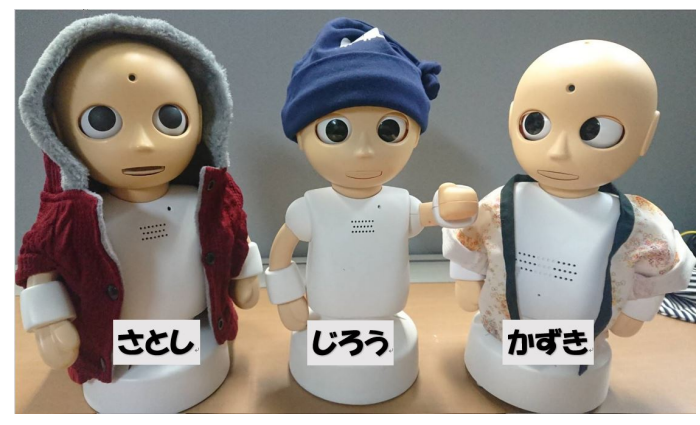

Fig. 4. Photograph shown to subject before second dialogue (each robot's name is displayed at the bottom)

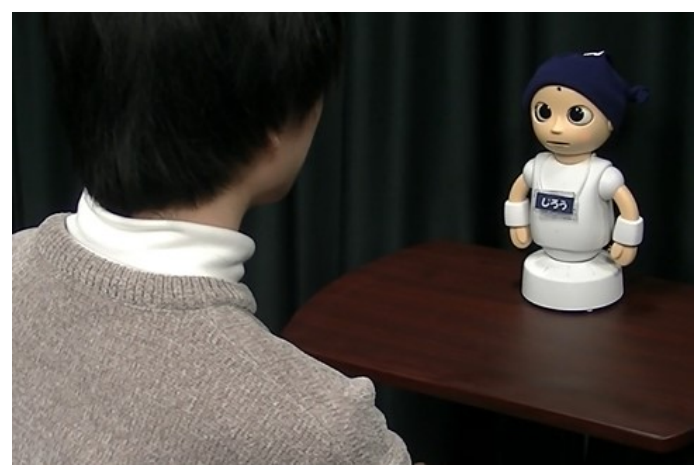

Fig. 5. Scene of experiment

of conversation, we evaluated the credibility of the experience mentioned by the robots [16], mind perception scale [17] and level of empathetic understanding [18]. We also evaluated the intention to use [19], which should increase with the improvement in the sense of conversation. If the credibility of the experience mentioned by the robots is high, a robot's personal statement such as "I like cake" can easily be accepted by the user. In this study, as the credibility of the experience mentioned by robots, we evaluated the perceived ability of the robot to judge the taste of food by asking "Do you think the robot can judge the taste of food?" Note that we labeled this question item as a mentioned experience for the sake of convenience. The mind perception scale can measure how humans attribute a mind to agents, and it consists of the experience and agency. For the experience, we evaluated the robot's capacity for pleasure, fear, and desire. For the agency, we evaluated the robot's capacity for memory, morality, and self-control. The level of empathetic understanding indicated whether the robot understands and feels empathy for the user's utterance, and it consisted of five similar questions, such as "Do you think the robot understood not only your words but also your intention?" or "Do you think the robot correctly understood what you said?". We labeled these five questions regarding empathy as empathy 1 to 5 . The intention to use indicated the extent to which the user would wish to talk to the robot again, and it consisted of three similar questions.

Following each conversation, the subject answered questions using the Likert scale. Furthermore, at the end of the experiment, he/she answered the same questions in a binary comparison instead of the Likert scale. It should be noted that, for the intention to use in the binary comparison, instead of using three similar questions, we used only one question, namely "I'm thinking of talking to the robot the next few days".

\section{E. Result}

Firstly, we had to verify whether a situation had occurred in which no disagreement took place between the robot and subject to determine the data used for analysis. This is because, if such a situation had occurred in the social condition, the robot would lose the opportunity to mention another robot; that is, the contents of the dialogue in the social condition would be almost the same as those in the non-social condition and we would need to exclude the data. Fig. 6 presents a histogram indicating the number of agreements in the dialogue under each condition. It can be observed from the figure that no situation occurred in which the robot agreed with the user all the time (among six times) in the dialogue under the social condition, that is, where the robot did not mention another robot at all. Therefore, we used and analyzed all of the subject data.

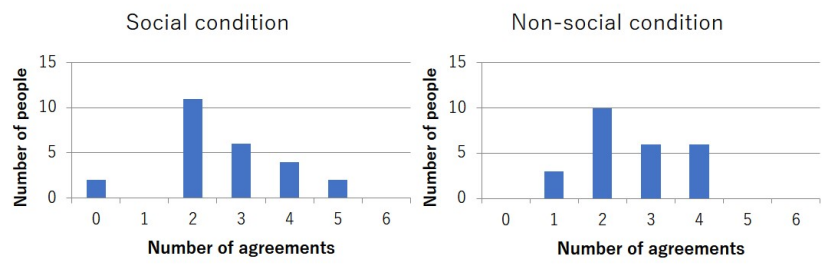

Fig. 6. Number of agreements

Table IV presents the box plot results of each question item evaluated on the Likert scale. We conducted a nonparametric test (the Wilcoxon signed-rank test) for each case. The test results demonstrated that the increase in the score of the agency was significantly higher in the social condition ( $M=3.41, S D=0.84)$ than in the non-social condition $(M=2.96, S D=0.77)$ with $Z=2.58, n=25, p=$ 0.0079 and $r=0.51$. However, we could not confirm the significant differences in the other question items.

TABLE IV

EVALUATION OF THE LIKERT SCALE $(* *: p<0.01)$

\begin{tabular}{lccccc}
\hline & \multicolumn{2}{c}{ Social condition } & \multicolumn{3}{l}{ Non-social condition } \\
\hline & Mean & SD & Mean & SD & $p$-value \\
\hline $\begin{array}{l}\text { Mentioned ex- } \\
\text { perience }\end{array}$ & 3.60 & 1.73 & 3.24 & 1.69 & 0.4336 \\
Experience & 2.80 & 0.79 & 2.80 & 0.79 & 0.9756 \\
Agency** & 3.41 & 0.84 & 2.96 & 0.77 & 0.0079 \\
Empathy & 1.46 & 0.64 & 1.33 & 0.60 & 0.1716 \\
Intention to use & 3.09 & 1.27 & 2.89 & 1.31 & 0.2212 \\
\hline
\end{tabular}

Fig. 7 presents the results of the evaluation of the binary comparison between the two conditions. We conducted the $\chi$-square test for each question item. We used the significance 
level adjusted for each of the five question items (mentioned experience, agency, experience, empathy and intention to use) by means of Bonferroni-correction. In particular, the adjusted significance levels were $\alpha^{\prime}=0.017$ for the experience and agency, $\alpha^{\prime}=0.010$ for the level of empathetic understanding, and $\alpha^{\prime}=0.050$ for the mentioned experience and intention to use (the same as before the adjustment). As a result, we could confirm significant differences in terms of empathy $1\left(\chi^{2}(1)=9.00, p=0.0027<0.010, \varphi=0.60\right)$, empathy $3\left(\chi^{2}(1)=6.76, p=0.0093<0.010, \varphi=0.52\right)$, empathy $5\left(\chi^{2}(1)=9.00, p=0.0027<0.010, \varphi=\right.$ $0.60)$, and intention to use $\left(\chi^{2}(1)=6.76, p=0.0093<\right.$ $0.050, \varphi=0.52)$. Moreover, we confirmed marginally significant differences in terms of empathy $2\left(\chi^{2}(1)=\right.$ $4.84, p=0.0278<0.050, \varphi=0.44)$ and empathy 4 $\left(\chi^{2}(1)=4.84, p=0.0278<0.050, \varphi=0.44\right)$.

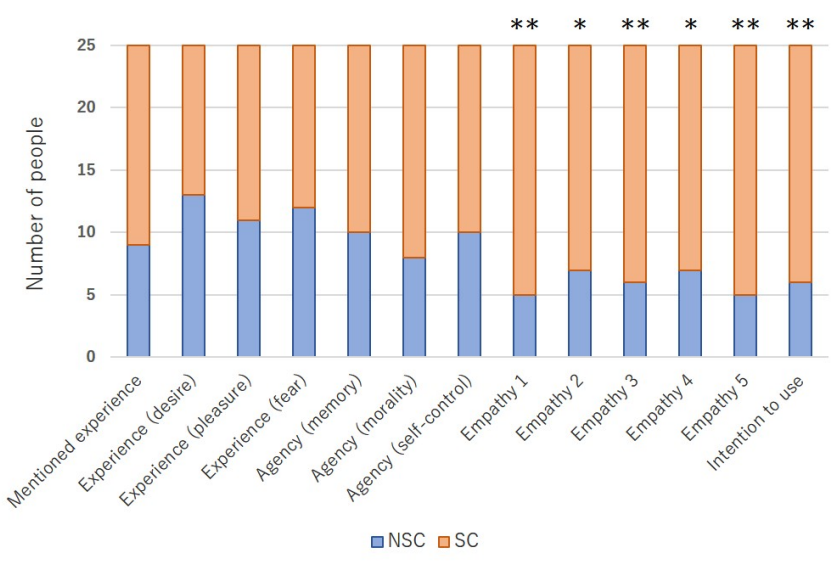

Fig. 7. Evaluation of binary comparison between two conditions ( $* *$ : $p<0.01, *: p<0.05)$

\section{DISCUSSION}

Firstly, we discuss why the significant difference for the agency was confirmed only in the evaluation of the Likert scale, whereas it could not be confirmed in that of the binary comparison. This may be because the number of people who felt higher levels of agency in the social condition was not large, but such people gave the agency a very high score on the Likert scale compared to that in the non-social condition.

Next, we discuss why the significant differences for the level of empathetic understanding and intention to use were confirmed only in the evaluation of the binary comparison, whereas it could not be confirmed in the evaluation of the Likert scale. This may be because, although many subjects had a positive impression under the social condition, the absolute difference in the impression of the robot in terms of these measurements was not sufficiently large to appear in the difference in the scores on the Likert scale.

Finally, we discuss why the significant differences for the credibility of the experience mentioned by the robots and the experience in mind perception were not confirmed in the evaluation of either the Likert scale or binary comparison. These question items focused on the robots' capabilities to have a subjective experience, such as the ability to judge the taste of food and capacity for fear. Sytsma et al. reported that humans tend not to attribute subjective experience (such as feeling pain or anger) to robots [20]. Therefore, it was difficult to improve the evaluation thereof significantly, as opposed to the agency, degree of empathetic understanding and intention to use.

\section{Number of agreements}

Several researchers have reported that people tend to react negatively when many disagreements occur during dialogue [5], [14]. Therefore, it was possible that the number of agreements in the dialogue would influence the results. For this reason, we first examined whether bias existed among the conditions in the number of agreements. We conducted a parametric test (the $t$-test). The test results did not demonstrate a significant difference in the number of agreements between the social condition $(M=2.64, S D=1.25)$ and non-social condition $(M=2.60, S D=1.00)$, with $t(24)=$ $-0.10507, p=0.9172$. Moreover, the mean difference in the number of agreements was only 0.04. Next, we examined whether correlations appeared between the number of agreements and the evaluations. Table $\mathrm{V}$ presents the correlation coefficients of the evaluations of each question on the Likert scale and $p$-values determined by the test of no correlation. For each question, the correlation coefficients were in the range of -0.2 to 0.2 , indicating little correlation. Moreover, no significant correlations were exhibited for all questions in the Likert scale evaluation in both conditions. Table VI presents the correlation coefficients of the evaluations on the binary comparison and $p$-values determined by the test of no correlation. Almost all of the correlation coefficients were in the range of -0.2 to 0.2 , and no significant correlations were exhibited for any questions in the binary comparison evaluation.

In conclusion, the significant differences confirmed in the experiment were not a result of the number of agreements, but were likely owing to the differences between the two conditions.

TABLE V

CORRELATIONS BETWEEN NUMBER OF AGREEMENTS AND EVALUATIONS ON LIKERT SCALE

\begin{tabular}{lrrrr}
\hline & \multicolumn{2}{c}{ Social condition } & \multicolumn{2}{c}{ Non-social condition } \\
\hline Question items & $\begin{array}{l}\text { Correlation } \\
\text { coefficients }\end{array}$ & $p$-value & $\begin{array}{l}\text { Correlation } \\
\text { coefficients }\end{array}$ & $p$-value \\
\hline $\begin{array}{l}\text { Mentioned expe- } \\
\text { rience }\end{array}$ & -0.0307 & 0.8842 & 0.0345 & 0.8699 \\
Experience & -0.0614 & 0.7706 & -0.0525 & 0.8031 \\
Agency & 0.0548 & 0.7947 & 0.1224 & 0.5600 \\
Empathy & 0.0157 & 0.9405 & 0.0056 & 0.9790 \\
Intention to use & -0.1090 & 0.6039 & 0.0930 & 0.6585 \\
\hline
\end{tabular}

\section{Limitations and future work}

In this experiment, the difference between the two conditions was the presence or absence of the statement regarding another robot. Although the robots showed concern to the subjects even in the non-social condition by saying "Don't 
TABLE VI

CORRELATIONS BETWEEN NUMBER OF AGREEMENTS AND EVALUATIONS ON BINARY COMPARISON

\begin{tabular}{lrc}
\hline Question items & $\begin{array}{r}\text { Correlation } \\
\text { coefficients }\end{array}$ & $p$-value \\
\hline Mentioned experience & 0.0680 & 0.7466 \\
Experience (desire) & -0.0327 & 0.8767 \\
Experience (pleasure) & -0.2303 & 0.2681 \\
Experience (fear) & 0.0327 & 0.8767 \\
Agency (memory) & 0.1667 & 0.4259 \\
Agency (morality) & 0.3150 & 0.1250 \\
Agency (self-control) & 0.0000 & 1.0000 \\
Empathy 1 & 0.2041 & 0.3277 \\
Empathy 2 & 0.0364 & 0.8630 \\
Empathy 3 & -0.0765 & 0.7164 \\
Empathy 4 & 0.0364 & 0.8630 \\
Empathy 5 & 0.2041 & 0.3277 \\
Intention to use & 0.1147 & 0.5851 \\
\hline
\end{tabular}

worry", the experimental design did not sufficiently address the fact that the quantity of the robot utterances differed between the conditions. Hence, we cannot fully deny the possibility that the subjects rated the social condition higher because there were more statements in the social condition. Moreover, the dialogue scenario in this experiment was only concerned with food preferences, so it is not clear what effect our dialogue strategy will have on other topics. Therefore, further investigation is required.

Furthermore, we only tested the effect of short-term interaction with subjects in the laboratory, and we therefore need to verify whether this dialogue strategy is useful in a real environment in the long run.

\section{CONCLUSIONS}

We have proposed a novel dialogue strategy whereby a robot mentions another robot in the form of gossiping. This dialogue strategy can improve the sense of conversation while avoiding privacy issues.

We examined the proposed strategy by conducting a conversation experiment evaluated by the subject impressions. The results demonstrated that the strategy could help the robot to obtain higher evaluations. In particular, the perceived mind was improved in the Likert scale evaluation, whereas the robot empathy and intention to use were improved in the binary comparison evaluation. Therefore, our dialogue strategy could contribute to the factors relating to the sense of conversation.

In the future, in addition to improving the experimental design, we will perform further verification and investigate the usefulness of this dialogue strategy when it is applied in a real environment.

\section{ACKNOWLEDGMENT}

This work was supported by JSPS KAKENHI Grant Number JP19H05691 and JST ERATO, Japan.

\section{REFERENCES}

[1] Gockley, R., A. Bruce, J. Forlizzi, M. Michalowski, A. Mundell, S. Rosenthal, B. Sellner, et al. 2005. Designing Robots for Long-Term Social Interaction. In 2005 IEEE/RSJ International Conference on Intelligent Robots and Systems, 133843. ieeexplore.ieee.org.

[2] Broekens, J., M. Heerink, H. Rosendal, et al. 2009. Assistive Social Robots in Elderly Care: A Review. Gerontechnology: International Journal on the Fundamental Aspects of Technology to Serve the Ageing Society 8 (2): 94103.

[3] Kanda, T., R. Sato, N. Saiwaki, and H. Ishiguro. 2007. A Two-Month Field Trial in an Elementary School for Long-Term Human-Robot Interaction. IEEE Transactions on Robotics 23 (5): 96271.

[4] Higashinaka, R., K. Sakai, H. Sugiyama, H. Narimatsu, T. Arimoto, T. Fukutomi, K. Matsui, et al. 2017. Argumentative Dialogue System Based on Argumentation Structures. SEMDIAL 2017 SaarDial, 154155.

[5] Uchida, T., T. Minato, and H. Ishiguro. 2016. A Values-Based Dialogue Strategy to Build Motivation for Conversation with Autonomous Conversational Robots. In 2016 25th IEEE International Symposium on Robot and Human Interactive Communication (RO-MAN), 20611. ieeexplore.ieee.org.

[6] Salter, T., K. Dautenhahn, and R. Bockhorst. 2004. Robots Moving out of the Laboratory - Detecting Interaction Levels and Human Contact in Noisy School Environments. In RO-MAN 2004. 13th IEEE International Workshop on Robot and Human Interactive Communication (IEEE Catalog No.04TH8759), 56368. ieeexplore.ieee.org.

[7] Leite, I., C. Martinho, A. Pereira, and A. Paiva. 2009. As Time Goes by: Long-Term Evaluation of Social Presence in Robotic Companions. In RO-MAN 2009 - The 18th IEEE International Symposium on Robot and Human Interactive Communication, 66974. ieeexplore.ieee.org.

[8] Fernaeus, Y., M. Hakansson, M. Jacobsson, and S. Ljungblad. 2010. How Do You Play with a Robotic Toy Animal? A Long-Term Study of Pleo. In Proceedings of the 9th International Conference on Interaction Design and Children, 3948. dl.acm.org.

[9] Kanda, T., M. Shiomi, Z. Miyashita, H. Ishiguro, and N. Hagita. 2010. A Communication Robot in a Shopping Mall. IEEE Transactions on Robotics 26 (5): 897913.

[10] Lee, M. K., J. Forlizzi, S. Kiesler, P. Rybski, J. Antanitis, and S. Savetsila. 2012. Personalization in HRI: A Longitudinal Field Experiment. In 2012 7th ACM/IEEE International Conference on Human-Robot Interaction (HRI), 31926. ieeexplore.ieee.org.

[11] Mahzoon, H., K. Ogawa, Y. Yoshikawa, M. Tanaka, K. Ogawa, R. Miyazaki, Y. Ota, and H. Ishiguro. 2019. Effect of Self-Representation of Interaction History by the Robot on Perceptions of Mind and Positive Relationship: A Case Study on a Home-Use Robot. Advanced Robotics: The International Journal of the Robotics Society of Japan 33 (21): 111228.

[12] Changzeng, F., Y. Yoshikawa, T. Iio, and H. Ishiguro. 2020. Sharing Experiences to Help a Robot Present Its Mind and Sociability. International Journal of Social Robotics.

[13] Goldfarb, A., and C. Tucker. 2012. Shifts in Privacy Concerns. The American Economic Review 102 (3): 34953.

[14] Benjafield, J., and J. Adams-Webber. 1976. THE GOLDEN SECTION HYPOTHESIS. British Journal of Psychology 67 (1): 1115.

[15] Yoshikawa, Y., T. Iio, T. Arimoto, H. Sugiyama, and H. Ishiguro. 2017. Proactive Conversation between Multiple Robots to Improve the Sense of Human-Robot Conversation. In 2017 AAAI Fall Symposium Series. aaai.org.

[16] Uchida, T., T. Minato, and H. Ishiguro. 2019. The relationship between Dialogue Motivation and Attribution of Subjective Opinions to Conversational Androids Transactions of the Japanese Society for Artificial Intelligence 34 (1): B - I62_18. (in Japanese)

[17] Gray, M., K. Gray, and D. Wegner. 2007. Dimensions of Mind Perception. Science 315 (5812): 619.

[18] Mishima, N., H. Shinkoda, H. Hukumoto. 1999. Kaigo ni ikasu sekkyokuteki keichohou [Actively listening for nursing care]. Medicus Shuppan Publisshers. (in Japanese)

[19] Heerink, M., B. Krose, V. Evers, and B. Wielinga. 2008. The Influence of Social Presence on Enjoyment and Intention to Use of a Robot and Screen Agent by Elderly Users. In RO-MAN 2008 - The 17th IEEE International Symposium on Robot and Human Interactive Communication, 695700. ieeexplore.ieee.org.

[20] Sytsma, J., and E. Machery. 2010. Two Conceptions of Subjective Experience. Philosophical Studies 151 (2): 299327. 